University of Nebraska - Lincoln

DigitalCommons@University of Nebraska - Lincoln

\title{
$4-2011$
}

\section{Efficiency enhancement in organic solar cells with ferroelectric polymers}

\author{
Yongbo Yuan \\ University of Nebraska-Lincoln, yyuan2@unl.edu \\ Timothy J. Reece \\ University of Nebraska-Lincoln, reecetj@unk.edu \\ Pankaj Sharma \\ University of Nebraska-Lincoln, psharma@huskers.unl.edu \\ Shashi Poddar \\ University of Nebraska-Lincoln \\ Stephen Ducharme \\ University of Nebraska-Lincoln, sducharme1@unl.edu \\ See next page for additional authors
}

Follow this and additional works at: https://digitalcommons.unl.edu/physicsducharme

Part of the Physics Commons

Yuan, Yongbo; Reece, Timothy J.; Sharma, Pankaj; Poddar, Shashi; Ducharme, Stephen; Gruverman, Alexei; Yang, Yang; and Huang, Jinsong, "Efficiency enhancement in organic solar cells with ferroelectric polymers" (2011). Stephen Ducharme Publications. 75.

https://digitalcommons.unl.edu/physicsducharme/75

This Article is brought to you for free and open access by the Research Papers in Physics and Astronomy at DigitalCommons@University of Nebraska - Lincoln. It has been accepted for inclusion in Stephen Ducharme Publications by an authorized administrator of DigitalCommons@University of Nebraska - Lincoln. 


\section{Authors}

Yongbo Yuan, Timothy J. Reece, Pankaj Sharma, Shashi Poddar, Stephen Ducharme, Alexei Gruverman, Yang Yang, and Jinsong Huang 
Published in Nature Materials 10 (APRIL 20I I); pp. 296-302; doi: I0.1038/NMAT295 I

Copyright (C) 201 I Macmillan Publishers Limited. www.nature.com/naturematerials Used by permission.

Submitted June 7, 2010 ; accepted December 23, 2010 ; published online February I3, $201 \mathrm{I}$.

\title{
Efficiency enhancement in organic solar cells with ferroelectric polymers
}

\author{
Yongbo Yuan, ${ }^{1,2}$ Timothy J. Reece, ${ }^{2,3}$ Pankaj Sharma, ${ }^{2,3}$ Shashi Poddar, ${ }^{2,3}$ \\ Stephen Ducharme, ${ }^{2,3}$ Alexei Gruverman, ${ }^{2,3}$ Yang Yang, ${ }^{4}$ and jinsong Huang ${ }^{1,2}$ \\ I. Department of Mechanical Engineering, University of Nebraska-Lincoln, Lincoln, Nebraska 68588-0656, USA \\ 2. Nebraska Center for Materials and Nanoscience, University of Nebraska-Lincoln, Lincoln, Nebraska 68583-0298, USA \\ 3. Department of Physics and Astronomy, University of Nebraska-Lincoln, Lincoln, Nebraska 68588-0299, USA \\ 4. Department of Materials Science and Engineering, University of California-Los Angeles, Los Angeles, California 90095-1595, USA \\ Corresponding author - J. Huang, email jhuang2@unl.edu
}

\begin{abstract}
The recombination of electrons and holes in semiconducting polymer-fullerene blends has been identified as a main cause of energy loss in organic photovoltaic devices. Generally, an external bias voltage is required to efficiently separate the electrons and holes and thus prevent their recombination. Here we show that a large, permanent, internal electric field can be ensured by incorporating a ferroelectric polymer layer into the device, which eliminates the need for an external bias. The electric field, of the order of $50 \mathrm{~V} \mathrm{~mm}^{-1}$, potentially induced by the ferroelectric layer is tens of times larger than that achievable by the use of electrodes with different work functions. We show that ferroelectric polymer layers enhanced the efficiency of several types of organic photovoltaic device from I-2\% without layers to $4-5 \%$ with layers. These enhanced efficiencies are 10-20\% higher than those achieved by other methods, such as morphology and electrode workfunction optimization. The devices show the unique characteristics of ferroelectric photovoltaic devices with switchable diode polarity and tunable efficiency.
\end{abstract}

Polymeric organic photovoltaic (OPV) cells with polymer-fullerene bulk heterojunction are promising candidates for future low-cost, high-performance energy sources, owing to their low material and processing costs and mechanical flexibility. ${ }^{1,2}$ High efficiencies of $6-8 \%$ have been realized in polymeric OPVs by reducing both the optical bandgap and the highest occupied molecular orbital of the semiconducting polymers, and by optimizing the morphology of polymerfullerene blend film with thermal annealing and solvent annealing. ${ }^{3-9}$ Although further optimization of the bandgap and highest occupied molecular orbital level of the semiconducting polymer is possible, ${ }^{3}$ the path to increasing OPV efficiency to $15 \%$ must include recovery of significant energy loss even in the relatively high efficiency devices demonstrated so far. ${ }^{10-}$ 12 There are five main causes of reduced efficiency in OPV devices: energy level misalignment, insufficient light trapping and absorption, low exciton diffusion lengths, and non-radiative recombination of charges or chargetransfer excitons (CTEs), which consist of electrons at the acceptor and holes at the donor bound by Coulomb attraction, and low carrier mobilities. ${ }^{11,13}$ In many of the most efficient polymer-fullerene OPV devices, $50 \%$ or more of the energy loss is caused by the recombination of CTEs (Reference 11). For example, if we look at the most intensively studied material system, regioregular poly(3-hexylthiophene) (P3HT) and phenyl-C61-butyric acid methyl ester (PCBM) blend, the photocurrent at the maximum power output point is only $70 \%$ of what could be extracted by externally applying a large reverse bias voltage to the device. ${ }^{14}$ The purpose of the present work is to show how to achieve greater efficiency using a large internal electrical field provided by the permanent electrical polarization of a ferroelectric (FE) polymer layer. To understand how this innovation works, we need to understand how internal electric fields affect charge extraction efficiency.
As illustrated in Figure 1a, CTEs form right after the photoinduced electron transfer. The next step is to separate them and enable them to contribute to the photocurrent. CTEs can be treated as a precursors of free carriers, and their bandgap sets a maximum value for the open-circuit voltage $\left(V_{\text {oc }}\right.$, References 10,15-23). The CTEs can be lost by non-radiative recombination when the dissociation driving forces (temperature and electric field) are small. The non-radiative recombination of CTEs, of course, reduces the photocurrent. In addition, the non-radiative recombination of CTEs reduces the free charge concentration, and then reduces the quasi-Fermi-energy (or chemical-potential) difference between electrons and holes, resulting in a lower $V^{\mathrm{oc}}$, reducing the output power even further. A strong reduction of the non-radiative recombination is required to reduce the fundamental efficiency loss. ${ }^{11,12,24}$ Using the detailed balance theory, which was used to calculate the theoretical efficiency limit of a p-n junction solar cell in Reference 25, to treat a polymer-fullerene solar cell, a $V_{\text {oc }}$ gain of $0.3-0.5 \mathrm{~V}$ by eliminating the non-radiative recombination pathways was predicted in Reference 13.

The mechanism of the non-radiative recombination of CTEs is not well understood. ${ }^{13,24}$ Nevertheless, there is a clear mechanism for dissociating the CTEs, and thus preventing recombination by applying a strong electric field to draw the electron and hole apart. ${ }^{26-28}$ The dissociation efficiency of CTEs into free charge carriers depends on the magnitude of the local electric field, where the probability of dissociation can be described quantitatively by the Onsager-Braun model. ${ }^{29}$ The probability $(P)$ that a charge-transfer state is dissociated can be described by a field-dependent dissociation rate, $k_{\mathrm{D}^{\prime}}$ and a field-independent decay rate, $k_{\mathrm{F}}$, of the CTE (References 26-28).

$$
P(E)=\frac{k_{D}(E)}{k_{\mathrm{D}}(E)+k_{\mathrm{F}}}
$$




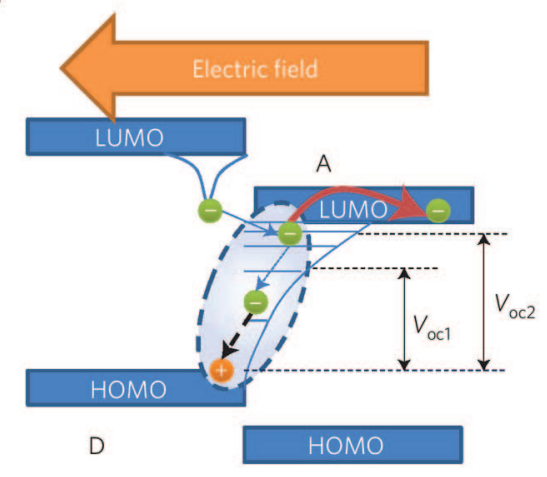

b

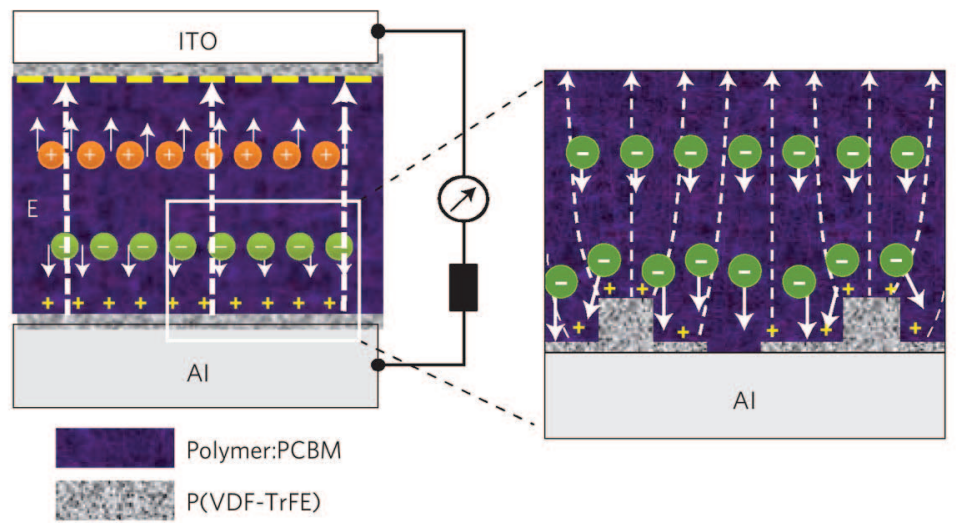

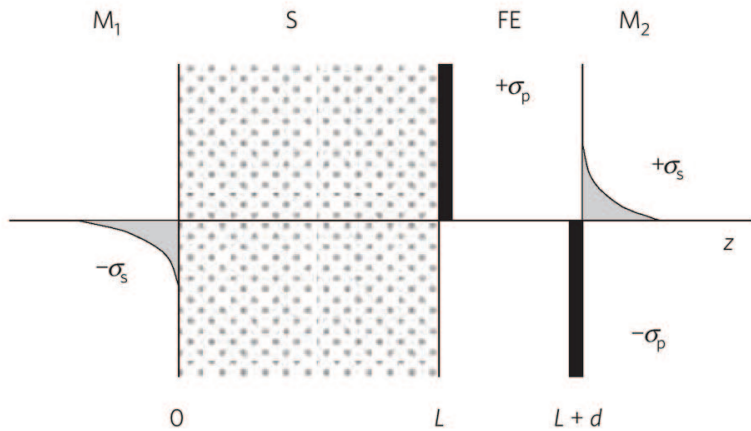

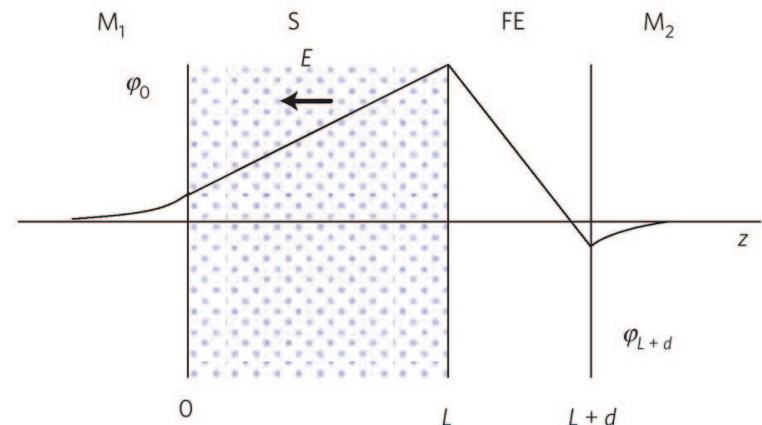

Figure I. Working principle of FE-OPV devices. a) Dynamic process for the dissociation of a (singlet) exciton to form a CTE (dashed oval) across the donoracceptor interface, and the dissociation of the CTE to free carriers. Reduced CTE recombination results in a higher electron quasi-Fermi level and thus a higher $V_{\text {oc }}$. b) The structure of polymer photovoltaic devices with FE interfacial layers and a schematic diagram of electric field induced by the polarized FE layer and the field-assisted charge extraction. The right-hand panel illustrates the electric-field distribution and electron conduction through the P(VDF-TrFE) on the Al side. c) \& d) Charge distribution and electrostatic potential profile of a junction with semiconductor (S) layer and ferroelectric (FE) layer inserted between two metal electrodes $(\mathrm{M} / \mathrm{S} / \mathrm{FE} / \mathrm{M})$ under the zero-bias condition. The surface charge density of the poled FE layer is $\sigma_{\mathrm{P}}$ The induced screening charge density at the two electrodes is $\sigma_{S}$.

$k_{\mathrm{D}}(E)$ is derived in Reference 28 to be

$$
k_{\mathrm{D}}(E)=k_{\mathrm{R}} \frac{3}{4 \pi a^{3}} \mathrm{e}^{-E \mathrm{~B} / k T}\left[1+b+\frac{b^{2}}{3}+\frac{b^{3}}{18}+\frac{b^{4}}{180}+\ldots\right]
$$

where $k_{\mathrm{R}}$ is the bimolecular rate constant of the bound e-h pair, $a$ is the initial separation of the bound e-h pair at the interface $\mathrm{CTE}, b=\mathrm{e}^{3} E / 8 \pi \varepsilon_{0} \varepsilon_{\mathrm{r}} k^{2} \mathrm{~T}^{2}, \varepsilon_{0}$ is the dielectric permittivity of the vacuum, $\varepsilon_{r}$ is the relative dielectric permittivity of the organic layer and $E_{\mathrm{B}}$ is the e-h pair's binding energy. In most polymerfullerene systems, a reverse bias larger than 5-10V (electric field of $25-50 \mathrm{~V} \mathrm{um}^{-1}$ for 200 -nm-thick film) is required to dissociate the majority of CTEs at room temperature. ${ }^{27} \mathrm{~A}$ small, internal electric field can be obtained even at zero device bias by using electrodes with different work functions. The electrode material used with conventional OPV devices, however, affords a workfunction difference of no more than $2 \mathrm{~V}$, producing an internal electric field of only $10 \mathrm{~V} \mathrm{~m}^{-1}$ for typical device dimensions, which is much too small for efficient CTE dissociation ${ }^{30}$. At the maximum-power point, the effective applied potential is even smaller, only $0.1-0.2 \mathrm{~V}$ (converted to 0.7 to $1.4 \mathrm{~V} \mathrm{\mu m}^{-1}$ for a polymer thickness of $150 \mathrm{~nm}$; References 14, 27). A new mechanism is then required to generated a ten-times-higher intrinsic electric field in the polymer semiconductor.

In this work, we incorporated the ultrathin FE film into polymer bulk heterojunction photovoltaic devices to increase efficiency with the induced polarization electric field. The device structure and working principle of the FE-OPV are shown in Figure 1b. The ultrathin FE layer(s) were inserted at the interfaces of the organic semiconductor and metal electrodes. The two-dimensional FE materials have a net polarization electric field after poling. With one type of charge partially or completely compensated by the metal electrode, the other type of charge sheet generates an electric field penetrating into the polymer semiconducting layer. The prerequisite for the operation of FE-OPV is the huge density of charges induced by FE polymer at the FE-semiconductor interface. These charges cannot be compensated by the low-concentration free charges in organic semiconductors, which leaves a large uncompensated internal field in the semiconductor. Vinylidene fluoride-trifluoroethylene copolymer, $\mathrm{P}(\mathrm{VDF}-\mathrm{TrFE})$ (Reference 31), was the initial choice for the FE material for its advantages of chemical inertness, low fabrication temperatures, photostability, and compatibility with polymer semiconducting materials. Its ferroelectricity originates from the molecular dipoles attached perpendicular to the chain axes and nearly normal to the plane of the thin film in the photovoltaic devices. A large electric polarization of the order of $100 \mathrm{mC} \mathrm{m}^{-2}$ can be produced with two-dimensional FE P(VDF-TrFE) films as thin as $1 \mathrm{~nm}$ (References 32-35). The light-absorbing material used for most of this study was P3HT:PC ${ }_{70} \mathrm{BM}$. Another low-bandgap polymer, poly[(4, 4'- bis(2-ethylhexyl)dithieno[3,2-b:2',3'-d] silole)-2,6-diyl-alt-(2,1,3-benzothiadiazole)-4,7-diyl] (PSBTBT) provided by Yang, ${ }^{36}$ was also briefly tested. The P(VDF-TrFE) thin film was deposited by Langmuir-Blodgett (LB) deposition or spin-coating. ${ }^{32,37}$ As $\mathrm{P}(\mathrm{VDF}-\mathrm{TrFE})$ is a good insulator, the thickness of P(VDF-TrFE) was controlled well below 10 $\mathrm{nm}$ so that carriers could tunnel through it to electrodes.

The maximum electric field induced in the semiconductor layer by the FE polymer film was calculated with a simplified model of internal electric potential balance at zero device bias 
(short-circuit condition). The model of the charge distribution is constructed by modifying the metal-FE-metal junction model of Reference 38 with a semiconductor layer inserted between the FE layer and one metal electrode. The charge distribution and respective electrostatic potential profile at short-circuit condition of a device with an FE layer inserted at the aluminiumsemiconductor interface are plotted in Figure 1c,d. A ThomasFermi model was used to describe the incomplete screening of the FE layer by the metal electrodes. The built-in electric field introduced by the P(VDF-TrFE) layer into the polymer is derived (see Supplementary Information for the derivation) to be

$$
E=\frac{d \sigma_{\mathrm{P}}}{\varepsilon_{0} \varepsilon_{\mathrm{FE}} L}
$$

where $\sigma_{\mathrm{P}}$ is the polarization charge density, $d$ is the thickness of the P(VDF-TrFE) layer, $L$ is the thickness of the polymer semiconductor layer and $\varepsilon_{\mathrm{FE}}$ is the relative dielectric constant of the $\mathrm{P}(\mathrm{VDF}-\mathrm{TrFE})$ layer. The underlying physics is that the ultrathin FE layer has a large surface charge density that raises the electric static potential at the surface of the semiconductor layer. The FE polymer is assumed to uniformly cover the whole surface and be polarized in a direction nearly perpendicular to the plane. Using the nominal remnant polarization of $100 \mathrm{mC} \mathrm{m}^{-2}$ for $\mathrm{P}(\mathrm{VDF}-\mathrm{TrFE})$, the electric field induced by the three monolayers (ML) (5 nm) P(VDF-TrFE) in a 150nm P3HT:PC ${ }_{70} \mathrm{BM}$ layer is calculated to be approximately $50 \mathrm{~V}$ $\mu^{-1}$. Therefore, 3ML of FE P(VDF-TrFE) can induce an electric field in the semiconductor layer that is sufficient to dissociate all the CTEs and suppress their non-radiative recombination. However, the actual induced electric field might be less than the model predicts owing to factors such as charge screening, possible incomplete poling of the FE layer and in particular the morphology of the FE layer, which will be shown below and in the supplementary document.

The influence of the induced electric field on the device performance is evident in the FE-OPV devices with P3HT:PC ${ }_{70} \mathrm{BM}$ as the active layer, as shown in Figure 2a. The addition of a 1ML LB film of P(VDF-TrFE) led to a small increase in short-circuit current $\left(I_{\mathrm{sc}}\right)$, even before poling, probably owing to the partial poling of the P(VDF-TrFE) LB films that typically occurs during the deposition process, ${ }^{47}$ which is confirmed by the piezoresponse force microscopy (PFM) study to be shown later. After poling the FE layer by applying a large positive voltage pulse on the $\mathrm{Al}$ electrode, an extra electric field was added to the polymer-fullerene layer which had the same direction as the electric field induced by the electrode work-function difference. After positive poling, the $I_{\mathrm{sc}}$ increased from approximately $10 \pm 1$ to $12 \pm 1 \mathrm{~mA} \mathrm{~cm}^{-2}$. There is a small variation in the maximum $I_{\mathrm{sc}}$ caused by the different $\mathrm{P} 3 \mathrm{HT}: \mathrm{PC}_{70} \mathrm{BM}$ thicknesses used and the variation of remanent $\mathrm{P}(\mathrm{VDF}-\mathrm{TrFE})$ polarization under different experimental conditions.

The electric field induced by the FE layer increases the $V_{\text {oc }}$ of the OPV devices as well. The increase of the $V_{\mathrm{oc}}$ can be understood by considering the photovoltage loss mechanisms in OPV devices. First, the above-mentioned, field-assisted CTE dissociation process increases the photocurrent generation by reducing the recombination and, in the meantime, preserves the high quasi-Fermi energy of electrons, which then contributes to increasing the $V_{\text {oc }}$. In this scenario, the $V_{\text {oc }}$ can be tuned continuously by controlling the electric field in the semiconductor, which has been realized by a partial polarization of $\mathrm{P}(\mathrm{VDF}-\mathrm{TrFE})$ in our devices (Supplementary Figure S2). Second, the voltage loss in photovoltaic devices is also related to the saturated dark current of the device. ${ }^{13,39,40}$ The poled FE polymer film was found to reduce the saturated dark current by two orders of magnitude, as shown in Figure $2 b$. The reduced saturated dark current should be ascribed to a high a

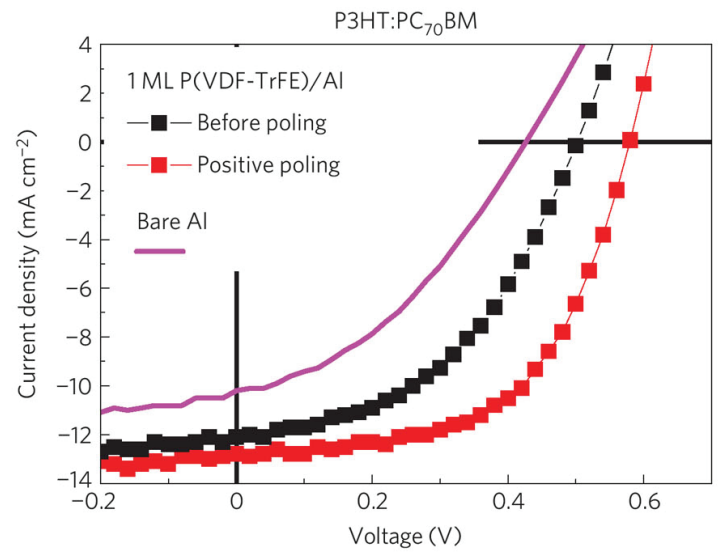

b

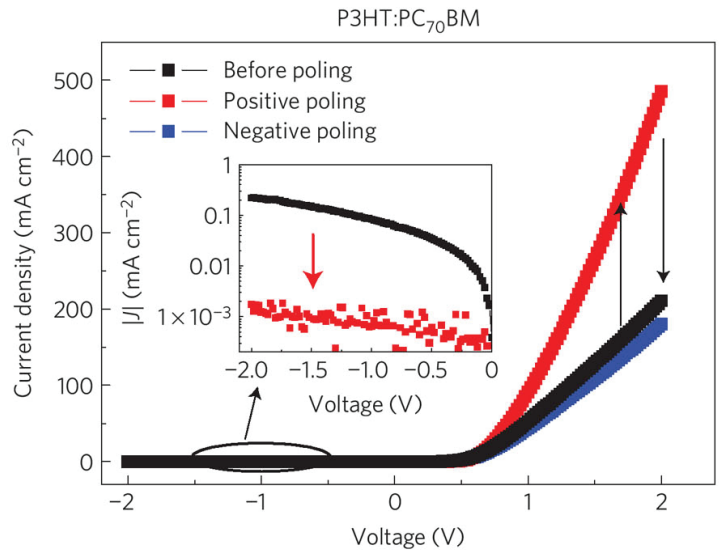

c

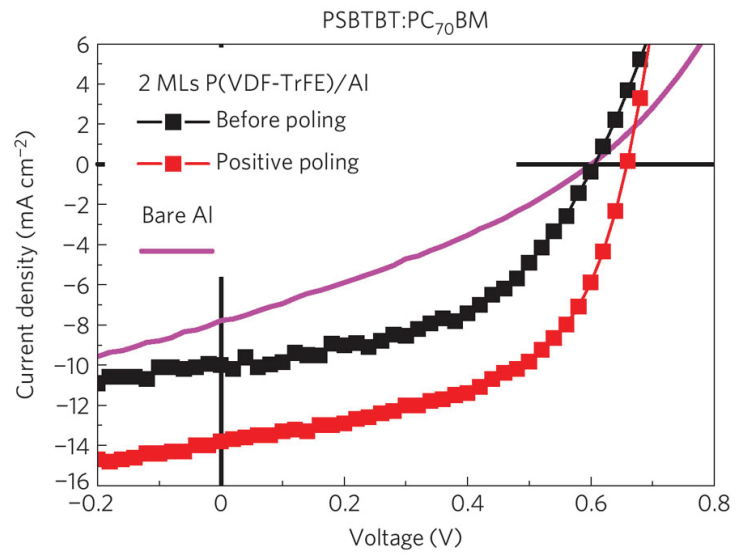

Figure 2. Improvement in device performance by the FE layer. The control devices have the structure of ITO/PEDOT:PSS/polymer:PC ${ }_{70} \mathrm{BM} / \mathrm{Al}$, and the $\mathrm{FE}-\mathrm{OPV}$ s have the $\mathrm{FE}$ layer inserted at the polymer/AI interfaces. a) Photocurrents of $\mathrm{P} 3 \mathrm{HT}: \mathrm{PC}_{70} \mathrm{BM}$ devices without an $\mathrm{FE}$ layer (magenta line) and with an FE layer before poling (black squares), and with an FE layer after positive poling (red squares). b) Dark current of devices with IML P(VDF-TrFE) film before and after positive and negative poling; the inset compares the reverse saturated dark currents of a device before and after positive poling. c) Photocurrent of a PSBTBT:PC ${ }_{70}$ BM device without an FE layer (magenta line), with an FE layer before poling (black square line) and with an FE layer after poling (red squares).

carrier-injection barrier at reverse bias introduced by the FE dipole layer. The poled FE polymer film also controls the dark current of the device. The energy efficiency is increased from $1.7 \%$ to $4.5 \%$ after positive poling.

In addition to the greater free-carrier generation by the increased geminate-pair separation, the induced electric field is also expected to increase the photogenerated-carrier collection by increasing the drift length of the carriers and/or extend the 

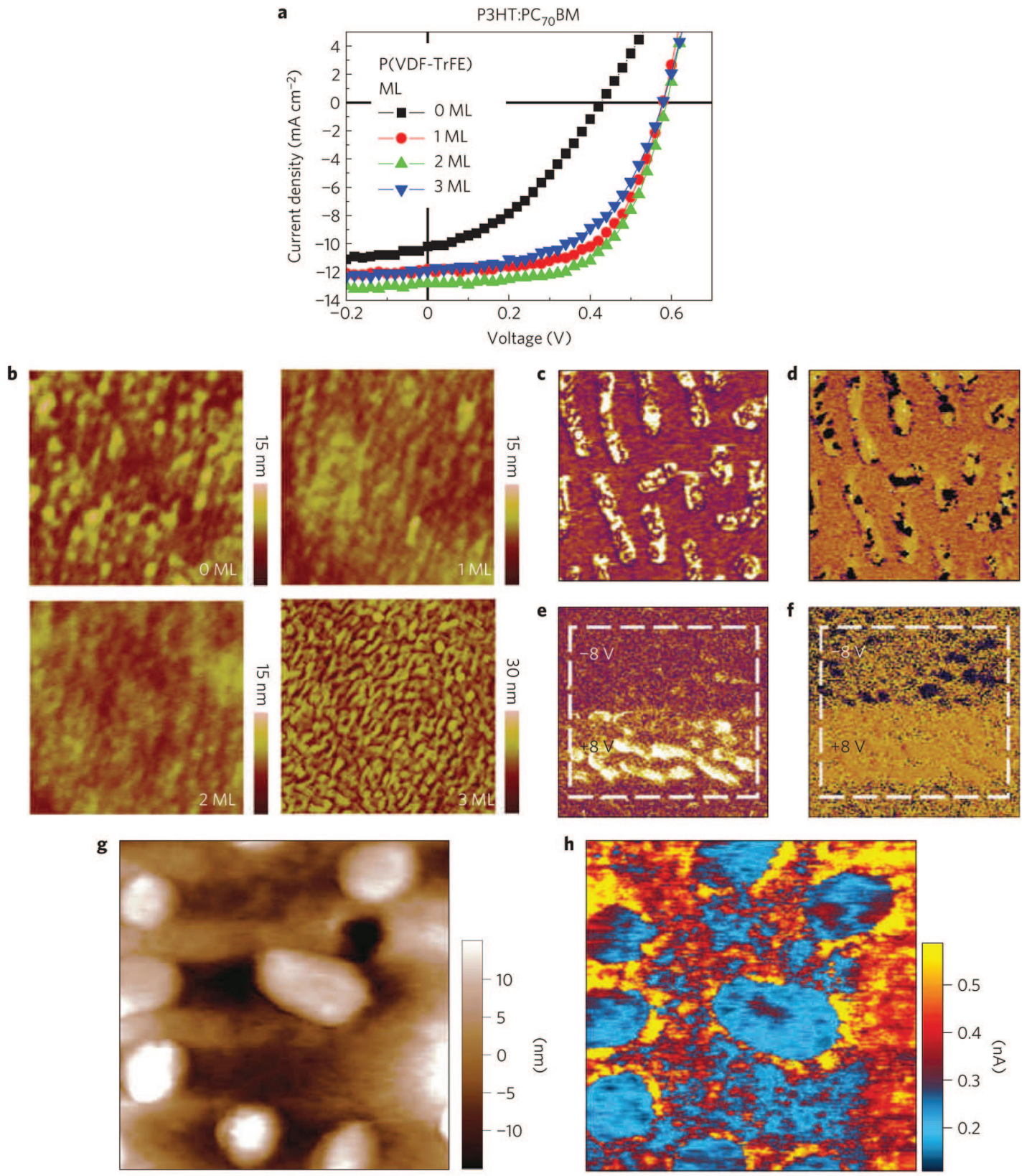

Figure 3. Morphologies of FE films and their effect on the device performance. a) Comparison of the $1-V$ curves of $P 3 \mathrm{HT}_{\mathrm{T}} \mathrm{PC} \mathrm{C}_{70} \mathrm{BM}$ devices with $0-3 \mathrm{ML}$ $\mathrm{P}(\mathrm{VDF}-\mathrm{TrFE})$ at the cathode interface. b)Surface morphology of P3HT:PCBM film covered by $0-3 \mathrm{ML}$ of P(VDF-TrFE) tested by AFM in tapping mode (4 $\mu \mathrm{m} \times$ $4 \mu \mathrm{m})$. c) \& d) PFM amplitude and phase image of a 2 ML P(VDF-TrFE) layer on a P3HT:PCBM film, tested by PFM in contact mode (I $\mu \mathrm{m} \times 1 \mu \mathrm{m})$ with Pt/Ticoated silicon tips. e) \& f) PFM amplitude and phase image of a 2ML P(VDF-TrFE) layer on a P3HT:PCBM top, poled by \pm 8V d.c. bias with a PFM tip under contact mode $(\mathrm{I} \mu \mathrm{m} \times \mu \mathrm{m})$. g) $\&$ h) AFM topography and conducting AFM current mapping of the same area $(500 \mathrm{~nm} \times 500 \mathrm{~nm})$ of a P3HT:PCBM film covered with $2 M L P(V D F-T r F E)$.

charge bimolecular-recombination lifetime. The carrier drift length is the product of carrier mobility, carrier recombination lifetime and internal electric field. Such a mechanism is especially important to semiconductor polymers with low carrier mobility, where a thicker layer can be used to increase the light absorption efficiency of OPV devices without sacrificing carrier collection efficiency. This was confirmed in our test of the low-bandgap material PSBTBT, which has a carrier mobility an order of magnitude lower than that of P3HT (Reference 7). It was found that a thicker $(90 \mathrm{~nm})$ film is needed to reach the optimized efficiency in the device with an FE layer than in a regular device $(80 \mathrm{~nm})$. The efficiency of the device with a PSBTBT:PC ${ }_{70} \mathrm{BM}$ active layer was increased from $1.5 \%$ to $4.9 \%$ by inserting $2 \mathrm{ML} \mathrm{P}(\mathrm{VDF}-\mathrm{TrFE})$ and poling (Figure 3c; Refer- ence 41). The devices with the Al cathode instead of calcium (Ca) were used as control devices because Ca chemically reacts with $\mathrm{P}(\mathrm{VDF}-\mathrm{TrFE})$ and kills the ferroelectricity of P(VDFTrFE). Nevertheless, all the FE-OPVs have already shown higher efficiencies than optimized devices with $\mathrm{Ca}$ as the cathode (see Table 1; References 42, 43).

According to Equation (3), a thicker P(VDF-TrFE) layer can introduce a larger electric field into the semiconductor layer. However, the increased P(VDF-TrFE) layer thickness also increases the contact resistance, which would reduce device efficiency. This was studied in our experiments by increasing the thickness of P(VDF-TrFE) from $0 \mathrm{ML}$ to $3 \mathrm{ML}$. The current-voltage $(I-V)$ characteristics of the $\mathrm{P} 3 \mathrm{HT}: \mathrm{PC}_{70} \mathrm{BM}$ devices with 0-3 ML of P(VDF-TrFE) after poling are shown in Fig- 
Table I. Comparing the device performance with 0-3 ML FE materials.

\begin{tabular}{|c|c|c|c|c|c|c|}
\hline Anode & Semiconducting layer & Cathode & $J_{s c}(m A ~ c m-2)$ & $V_{\text {oc }}(\mathrm{V})$ & $\mathrm{FF}(\%)$ & PCE (\%) \\
\hline \multirow[t]{3}{*}{$\mathrm{MoO}_{3} / \mathrm{Ag}$} & \multirow[t]{3}{*}{ P3HT:PC ${ }_{60} \mathrm{BM}$} & Bare ITO & 6.41 & 0.183 & 31 & 0.37 \\
\hline & & I ML LB film/ITO & 7.60 & 0.405 & 41 & 1.3 \\
\hline & & $2 \mathrm{ML}$ LB film/ITO & 7.49 & 0.460 & 49 & 1.7 \\
\hline \multirow[t]{3}{*}{ ITO/PEDOT:PSS } & \multirow{3}{*}{ P3HT:PC ${ }_{70} \mathrm{BM}$} & Bare Al & 10.2 & 0.426 & 38 & I.7 \\
\hline & & $\mathrm{Ca} / \mathrm{Al}$ & 10.5 & 0.582 & 61 & 3.7 \\
\hline & & I ML LB film/AI & 11.8 & 0.579 & 60 & 4.1 \\
\hline \multirow[t]{5}{*}{ ITO/PEDOT:PSS } & \multirow[t]{5}{*}{ PSBTBT:PC 70 BM } & Bare Al & 7.78 & 0.600 & 31 & 1.5 \\
\hline & & $\mathrm{Ca} / \mathrm{Al}$ & 13.2 & 0.660 & 51 & 4.4 \\
\hline & & I ML LB film/AI & 12.1 & 0.647 & 53 & 4.2 \\
\hline & & 2 ML LB film/Al & 13.8 & 0.660 & 54 & 4.9 \\
\hline & & 3 ML LB film/AI & 13.1 & 0.654 & 54 & 4.6 \\
\hline
\end{tabular}

ure 3a. One or two monolayers of $\mathrm{P}(\mathrm{VDF}-\mathrm{TrFE})$ was the optimum thickness range for $\mathrm{P} 3 \mathrm{HT}: \mathrm{PC}_{70} \mathrm{BM}$. From this point of view, the absence of a critical thickness for the ferroelectricity of two-dimensional $\mathrm{P}(\mathrm{VDF}-\mathrm{TrFE})$ is crucial to ensuring that the FE-OPV can work so well. An increased thickness to three monolayers of $\mathrm{P}(\mathrm{VDF}-\mathrm{TrFE})$ resulted in slightly reduced efficiency for P3HT:PCBM devices, although it is still much larger than the device without an FE layer. To understand the device performance variation with $\mathrm{P}(\mathrm{VDFTrFE})$ thickness, we studied the morphology of $\mathrm{P}(\mathrm{VDF}-\mathrm{TrFE})$ by atomic force microscopy (AFM), as shown in Figure $3 b$. The pristine P(VDF-TrFE) LB films are continuous before annealing but become non-continuous and rougher after thermal annealing. The roughness data, ranging from $0.8 \mathrm{~nm}$ to $1.7 \mathrm{~nm}$ for films before annealing, and from $1.0 \mathrm{~nm}$ to $3.6 \mathrm{~nm}$ after annealing, are summarized in Supplementary Table S1. It is clearly shown that the $3 \mathrm{ML}$ films of $\mathrm{P}(\mathrm{VDF}-\mathrm{TrFE})$ aggregate after annealing, forming many nanomesas. This must be caused by the mismatch of surface energy between the P3HT:PCBM film and P(VDF-TrFE) film, and the formation of nanomesas helps to minimize the surface energy. ${ }^{44}$ The thickness of the nanomesas formed by annealing $3 \mathrm{ML}$ of $\mathrm{P}(\mathrm{VDF}-\mathrm{TrFE})$ is approximately $10 \mathrm{~nm}$ (Supplementary Figure S3), which is too thick for efficient tunnelling of electrons through this layer, and explains the reduced photocurrent. The highest efficiency occurs at a balance of electric fields added by the $P(V D F-T r F E)$ layers and the series resistance introduced by the insulating $\mathrm{P}(\mathrm{VDF}-\mathrm{TrFE})$ layers.

The 1-2 ML of P(VDF-TrFE) film were also found to be noncontinuous, with $\mathrm{P}(\mathrm{VDF}-\mathrm{TrFE})$ islands sitting on P3HT:PCBM, from the PFM measurement (Figure 3c,d). The spontaneous polarization of $\mathrm{P}(\mathrm{VDF}-\mathrm{TrFE})$ on P3HT:PCBM exhibits a preferred direction and the same direction as positively poled $\mathrm{P}(\mathrm{VDF}-\mathrm{TrFE})$, which explains the observed increased photocurrent in the devices with P(VDF-TrFE) layers even before positive poling. The detailed mechanism of the initial net polarization is still not clear but is believed to be related to the difference in chemical affinity of $\mathrm{F}$ and $\mathrm{H}$ to the substrates or semiconducting polymer. ${ }^{45}$ The P(VDFTrFE) films on P3HT:PCBM were directly poled by the PFM tip with an applied voltage of $\pm 8 \mathrm{~V}$ and the polarization imaged by PFM (Figure $3 \mathrm{e}, \mathrm{f})$, which confirmed the ultrathin P(VDF-TrFE) film's ferroelectricity at the nanoscale. After poling, the electric fields from all FE film areas point in the same direction.

To understand how the current conducts through the ultrathin insulating FE films, their conductance was measured at nanoscale with conducting AFM in conjugation with PFM on the same area $(500 \mathrm{~nm} \times 500 \mathrm{~nm})$ of 2 ML P(VDF-TrFE)-cov- ered P3HT:PCBM/poly(3,4-ethylenedioxythiophene):polysty renesulphonate (PEDOT:PSS)/indium tin oxide (ITO) under small bias. The conductance of the P(VDF-TrFE) can be well correlated to the thickness and morphology. It is clear that the current is smaller through the nanomesa than through the area surrounding the nanomesa unless at FE domain boundaries. It is evident in Figure 3e,f that there is still a very thin layer of $\mathrm{P}(\mathrm{VDF}-\mathrm{TrFE})$ surrounding the nanomesas whose thickness might be in the range of tunnelling length. The thickness of this remaining layer was not determined because of the large roughness of the semiconducting polymer film. The charges can go through these thin $\mathrm{P}(\mathrm{VDF}-\mathrm{TrFE})$ layers although the electric field in P(VDF-TrFE) is in the opposite direction. It is believed that the thicker FE nanomesas mainly contribute a large induced electric field in the semiconductor polymer on the top of and around them and increase the average induced electric field, but thinner FE surrounding layers are more efficient in collecting charges. This result also implies the potential to further increase the FE-OPV efficiency by optimizing the morphology of FE polymer such as the thickness and the area ratio of thick and thin FE polymer islands.

The non-continuous $\mathrm{P}(\mathrm{VDF}-\mathrm{TrFE})$ nanomesas covered less than $50 \%$ of the surface of P3HT:PCBM film, as inferred from Figure $3 \mathrm{c}, \mathrm{d}$. The incomplete coverage inevitably leads to a smaller, induced internal electric field than calculated by Equation (3). The actual electric field produced by the FE layer was calculated by extrapolating the photocurrent of the device without P(VDF-TrFE) at reverse bias (Supplementary Figure S4). Here we used the assumption that the photocurrent is only determined by the effective electric field applied. ${ }^{27}$ The enhanced $I_{\mathrm{sc}}$ in FE-OPV compared with the regular OPV is attributed to the electric field added by the FE layer. The extracted electric field due to the FE polymer was approximately $12 \pm 3 \mathrm{~V}$ $\mu \mathrm{m}^{-1}$ added by P(VDF-TrFE), which is much less than the calculated result of $50 \mathrm{~V} \mathrm{\mu m}^{-1}$. There is therefore plenty of room for improvement, which was verified by the observation that application of a large reverse bias of $-5 \mathrm{~V}$ produced an even larger photocurrent of $15 \mathrm{~mA} \mathrm{~cm}^{-2}$ (compared with $10 \mathrm{~mA} \mathrm{~cm}^{-2}$ at the maximum-power point) in the $\mathrm{P} 3 \mathrm{HT}: \mathrm{PC}_{70} \mathrm{BM}$ device.

The FE-OPV shows the unique characteristics of an FE photovoltaic with switchable diode polarity and tunable device efficiencies. As shown in Figure 4, the polarity of the device with 1ML P(VDF-TrFE) inserted at both anode and cathode can be completely switched by the $\pm 15 \mathrm{~V}$ pulse. The current of the pristine device shows nearly Ohmic behavior and little rectification. The $I-V$ curve becomes asymmetric with a much larger rectification with diode characteristics after positive poling or 


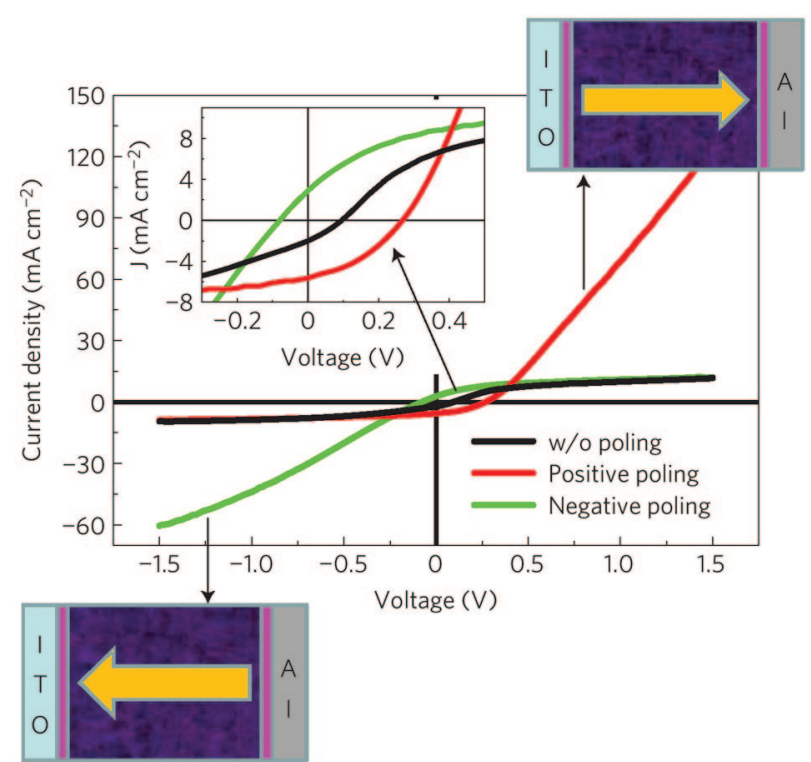

Figure 4. Switch behaviors of FE-OPV devices. Photocurrent output from a device with a structure of ITO/IML P(VDF-TrFE)/P3HT:PCBM/IML P(VDF$\operatorname{TrFE}$ )/Al. The inset zooms in the $I-V$ curves around zero bias.

negative poling. This is the first time that both a switchable diode and photovoltaic devices have been demonstrated. The larger $V_{\mathrm{oc}}$ in the positively poled devices is consistent with the fact that there is a built-in electric field pointing from $\mathrm{Al}$ to ITO as well as an asymmetric polarization of P(VDF-TrFE) due to the interface chemistry effect. ${ }^{46}$ The devices with an FE layer inserted at the cathode interface, either the $\mathrm{Al}$ cathode or the ITO cathode, also show a switchable photocurrent alternating between high-efficiency and low-efficiency states as the FE film polarization is switched back and forth by positive and negative voltage pulses (see Supplementary Figures S5 and S7). This result also demonstrates that $\mathrm{P}(\mathrm{VDF}-\mathrm{TrFE})$ can be used as a universal interface material for electrodes, greatly expanding the design and fabrication flexibility. The switchable nature of the photocurrent and photovoltage by poling $\mathrm{P}(\mathrm{VDF}-\mathrm{TrFE})$ along the two opposite directions implies that the FE polarization has a dominant role in the observed photovoltaic enhancement effect. The efficiency of our FE-OPV is several orders of magnitude larger than the largest reported efficiency of other FE photovoltaic devices. ${ }^{47}$

In summary, we report an FE-OPV hybrid device, which has both FE photovoltaic and OPV characteristics. The electric field induced by the ultrathin FE polymer films significantly increased the device efficiency, from 1-2\% without the FE film to $4-5 \%$ after poling the FE layer. These enhanced efficiencies are $10-20 \%$ higher than those achieved by other methods, such as morphology and electrode work-function optimization. There is still ample opportunity to further increase the efficiency of the FE-OPV devices with our current semiconducting polymers by using a better-controlled morphology and thickness of the FE film. The theoretical limit of $I_{\mathrm{sc}}$ from FEOPV can be derived from the photocurrent of regular OPV at a reverse bias of $-10 \mathrm{~V}$. With the increased electric field to eliminate the CTEs' recombination and bimolecular recombination, ${ }^{48}$ a further increase of $V_{\text {oc }}$ is expected. ${ }^{13}$ Also note that the increase of $V_{\mathrm{oc}}$ may not be as pronounced as the optimum 0.3$0.5 \mathrm{~V}$ because there may be modes of non-radiative charge recombination that are field independent, such as defect- or impurity-mediated charge recombination. The increased $V_{\text {oc }}$ can also be partially offset by the contact resistance brought by the insulating FE layer. Further work is necessary to optimize the morphology of the FE film to take full advantage of its electric field and increase charge-collection efficiencies, while ensuring efficient transfer to the electrodes with minimum conduction resistance through the FE film.

\section{Methods}

For the device fabrication, PEDOT:PSS (Baytron-P 4083) was first spin-cast onto a cleaned ITO/glass substrate at a spin speed of 3,500 r.p.m., which gives a PEDOT:PSS film thickness of approximately 30 $\mathrm{nm}$, as measured with a Dektek profilometer. The spun PEDOT:PSS was then baked at $120^{\circ} \mathrm{C}$ for $20 \mathrm{~min}$ before spin-casting the polymer film. P3HT (purchased from Rieke Metals, used as received) was first dissolved in 1,2-dichlorobenzene to make $30 \mathrm{mg} \mathrm{ml}^{-1}$ solution, followed by blending with $\mathrm{PC}_{70} \mathrm{BM}$ (purchased from Nano-C, used as received) in a ratio of 1:1 by weight. The blend was stirred for about 14 $\mathrm{h}$ at $40^{\circ} \mathrm{C}$ in a nitrogen-filled glove box. The active layer was obtained by spin-coating the blend at 800 r.p.m. for $20 \mathrm{~s}$, and the thickness of the P3HT:PC ${ }_{70} \mathrm{BM}$ film is approximately $150 \mathrm{~nm}$. For the devices based on PSBTBT, both PSBTBT and $\mathrm{PC}_{70} \mathrm{BM}$ were dissolved in chlorobenzene in a 1.5:1 $\left(10 \mathrm{mg} \mathrm{ml}^{-1}\right)$ ratio. The film was obtained by spin-coating at 2,000 r.p.m., then annealed at $140{ }^{\circ} \mathrm{C}$ for $5 \mathrm{~min}$, and the thickness of the film was approximately $90 \mathrm{~nm}$.

The LB deposition, although not the only choice for the deposition of P(VDF-TrFE) onto polymer semiconductor or ITO, has the unique capability to form a conformal and controlled ultrathin film to the level of $1 \mathrm{ML}$ and has more tolerance to surfaces with different surface energies. ${ }^{47}$ For the deposition of FE LB films, the random copolymer $\mathrm{P}(\mathrm{VDF}-\mathrm{TrFE})$, containing $70 \%$ vinylidene fluoride and 30\% trifluoroethylene, was dissolved in dimethyl sulphoxide with a concentration of $0.05 \%$ by weight. The polymer was dispersed on the surface of ultrapure $(18 \mathrm{M} \Omega \mathrm{cm})$ water, and slowly compressed to a surface pressure of $5 \mathrm{mN} \mathrm{m}^{-1}$ at room temperature in air. Then the LB layer was transferred to a glass/ITO substrate covered with P3HT:PC ${ }_{70} \mathrm{BM}$ or PSBTBT:PC ${ }_{70} \mathrm{BM}$ film. The $\mathrm{P}(\mathrm{VDF}-\mathrm{TrFE})$ films were deposited $1 \mathrm{ML}$ at a time by horizontal dipping. The samples were annealed at $135^{\circ} \mathrm{C}$ in $\mathrm{N}_{2}$ for half an hour to improve the crystallinity of the P(VDF-TrFE; Reference 30). The FE LB film preparation procedure is described in detail in Reference 48.

The active device area was $0.08 \mathrm{~cm}^{2}$. Photocurrent measurement was done under simulated air mass 1.5 global irradiation $(100 \mathrm{~mW}$ $\mathrm{cm}^{-2}$ ) using a xenon-lamp-based solar simulator (Oriel 67005, 150W Solar Simulator). A Schott visible-color glass-filtered (KG5 color-filtered) Si diode (Hamamatsu S1133) was used to calibrate the light intensity before photocurrent measurement.

\section{References}

1. Yu, G., Gao, J., Hummelen, J. C., Wudl, F. \& Heeger, A. J. Polymer photovoltaic cells: Enhanced efficiencies via a network of internal donor-acceptor heterojunctions. Science 270, 1789-1791 (1995).

2. Sariciftci, N. S., Smilowitz, L., Heeger, A. J. \& Wudl, F. Photoinduced electron transfer from a conducting polymer to buckminsterfullerene. Science 258, 1474-1476 (1992).

3. Chen, H. Y. et al. Polymer solar cells with enhanced open-circuit voltage and efficiency. Nature Photon. 3, 649-653 (2009).

4. Park, S. H. et al. Bulk heterojunction solar cells with internal quantum efficiency approaching 100\%. Nature Photon. 3, 297-303 (2009).

5. Liang, Y. et al. For the bright future-bulk heterojunction polymer solar cells with power conversion efficiency of 7.4\%. Adv. Mater. 22, 1-4 (2010).

6. Kim, J. Y. et al. Efficient tandem polymer solar cells fabricated by all-solution processing. Science 317, 222-225 (2007).

7. Chen, H-Y. et al. Silicon atom substitution enhances interchain packing in a thiophene-based polymer system. Adv. Mater. 21, 1-5 (2009).

8. Liang, Y. Y. et al. Development of new semiconducting polymers for high performance solar cells. J. Am. Chem. Soc. 131, 56-57 (2009).

9. Liang, Y. Y. et al. Highly efficient solar cell polymers developed via finetuning of structural and electronic properties. J. Am. Chem. Soc. 131, 7792-7799 (2009).

10. Veldman, D., Meskers, S. C. J. \& Janssen, R. A. J. The energy of chargetransfer states in electron donor-acceptor blends: Insight into the energy losses in organic solar cells. Adv. Funct. Mater. 19, 1939-1948 (2009). 
11. Kirchartz, T., Taretto, K. \& Rau, U. Efficiency limits of organic bulk heterojunction solar cells. J. Phys. Chem. C 113, 17958-17966 (2009).

12. Veldman, D. et al. Compositional and electric field dependence of the dissociation of charge transfer excitons in alternating polyfluorene copolymer/fullerene blends. J. Am. Chem. Soc. 130, 7721-7735 (2008).

13. Vandewal, K., Tvingstedt, K., Gadisa, A., Inganäs, O. \& Manca, J. V. On the origin of the open-circuit voltage of polymer-fullerene solar cells. Nature Mater. 8, 904-909 (2009).

14. Shrotriya, V., Yao, Y., Li, G. \& Yang, Y. Effect of self-organization in polymer/fullerene bulk heterojunctions on solar cell performance. Appl. Phys. Lett. 89, 063505 (2006).

15. Brabec, C. J. et al. Origin of the open circuit voltage of plastic solar cells. Adv. Funct. Mater. 11, 374-380 (2001).

16. Gadisa, A., Svensson, M., Andersson, M. R. \& Inganas, O. Correlation between oxidation potential and open-circuit voltage of composite solar cells based on blends of polythiophenes/fullerene derivative. Appl. Phys. Lett. 84, 1609-1611 (2004).

17. Cravino, A. Origin of the open circuit voltage of donor-acceptor solar cells: Do polaronic energy levels play a role? Appl. Phys. Lett. 91, 243502 (2007).

18. Cremer, J., Bauerle, P., Wienk, M. M. \& Janssen, R. A. J. High open-circuit voltage poly(ethynylene bithienylene): Fullerene solar cells. Chem. Mater. 18, 5832-5834 (2006).

19. Roquet, S. et al. Triphenylamine-thienylenevinylene hybrid systems with internal charge transfer as donor materials for heterojunction solar cells. J. Am. Chem. Soc. 128, 3459-3466 (2006).

20. Mutolo, K. L., Mayo, E. I., Rand, B. P., Forrest, S. R. \& Thompson, M. E. Enhanced open-circuit voltage in subphthalocyanine/C-60 organic photovoltaic cells. J. Am. Chem. Soc. 128, 8108-8109 (2006).

21. Scharber, M. C. et al. Design rules for donors in bulk-heterojunction solar cells-towards $10 \%$ energy-conversion efficiency. Adv. Mater. 18, 789-794 (2006).

22. Rand, B. P., Burk, D. P.\&Forrest, S. R. Offset energies at organic semiconductor heterojunctions and their influence on the open-circuit voltage of thin-film solar cells. Phys. Rev. B 75, 115327-115337 (2007).

23. Schueppel, R. et al. Optimizing organic photovoltaics using tailored heterojunctions: A photoinduced absorption study of oligothiophenes with low band gaps. Phys. Rev. B 77, 085311-085324 (2008).

24. Tvingstedt, K. et al. Electroluminescence from charge transfer states in polymer solar cells. J. Am. Chem. Soc. 131, 11819-11824 (2009).

25. Shockley, W. \& Queisser, H. Detailed balance limit of efficiency of p-n junction solar cells. J. Appl. Phys. 32, 510-519 (1961).

26. Veldman, D. et al. Compositional and electric field dependence of the dissociation of charge transfer excitons in alternating polyfluorene copolymer/fullerene blends. J. Am. Chem. Soc. 130, 7721-7735 (2008).

27. Mihailetchi, V. D., Koster, L. J. A., Hummelen, J. C. \& Blom, P. W. M. Photocurrent generation in polymer-fullerene bulk heterojunctions. Phys. Rev. Lett. 93, 216601-216604 (2004).

28. Braun, C. L. Electric field assisted dissociation of charge transfer states as a mechanism of photocarrier production. J. Chem. Phys. 80, 41574161 (1984).

29. Onsager, L. Deviations from ohm's law in weak electrolytes. J. Chem. Phys. 2, 599-615 (1934).

30. Kim, J. Y. et al. New architecture for high-efficiency polymer photovoltaic cells using solution-based titanium oxide as an optical spacer. Adv. Mater. 18, 572-576 (2006).

31. Furukawa, T. Ferroelectric properties of vinylidene fluoride copolymers. Phase Transit. 18, 143-211 (1989).

32. Bune, A. V. et al. Two-dimensional ferroelectric films. Nature 391, 874877 (1998).
33. Ducharme, S., Palto, S. P., Blinov, L. M. \& Fridkin, V. M. Physics of twodimensional ferroelectric polymers. AIP Conf. Proc. 535, 354-363 (2000).

34. Junquera, J. \& Ghosez, P. Critical thickness for ferroelectricity in perovskite ultrathin films. Nature 422, 506-509 (2003).

35. Ahn, C. H., Rabe, K. M. \& Triscone, J. M. Ferroelectricity at the nanoscale: Local polarization in oxide thin films and heterostructures. Science 303, 488-491 (2004).

36. Hou, J. H., Chen, H. Y., Zhang, S. Q., Li, G. \& Yang, Y. Synthesis, characterization, and photovoltaic properties of a low band gap polymer based on silole-containing polythiophenes and 2,1,3-benzothiadiazole. J. Am. Chem. Soc. 130, 16144-16145 (2008).

37. Sorokin, A. V., Bai, M., Ducharme, S. \& Poulsen, M. Langmuir-Blodgett films of polyethylene. J. Appl. Phys. 92, 5977-5981 (2002).

38. Zhuravlev, M. Y., Sabirianov, R. F., Jaswal, S. S. \& Tsymbal, E. Y. Giant electroresistance in ferroelectric tunnel junctions. Phys. Rev. Lett. 94, 246802 (2005).

39. Li, N., Lassiter, B. E., Lunt, R. R., Wei, G. \& Forrest, S. R. Open circuit voltage enhancement due to reduced dark current in small molecule photovoltaic cells. Appl. Phys. Lett. 94, 023307 (2009).

40. Potscavage, W. J., Yoo, S. \& Kippelen, B. Origin of the open-circuit voltage in multilayer heterojunction organic solar cells. Appl. Phys. Lett. 93, 193308 (2008)

41. Liang, Y. et al. Development of new semiconducting polymers for high performance solar cells. J. Am. Chem. Soc. 131, 56-57 (2008).

42. Sista, S., Hong, Z. R., Park, M. H., Xu, Z. \& Yang, Y. High-efficiency polymer tandem solar cells with three-terminal structure. Adv. Mater. 22, E77-E80 (2010).

43. Sista, S. et al. Highly efficient tandem polymer photovoltaic cells. Adv. Mater. 22, 380-383 (2010).

44. Bai, M. J. \& Ducharme, S. Ferroelectric nanomesa formation from polymer Langmuir-Blodgett films. Appl. Phys. Lett. 85, 3528-3530 (2004).

45. Duan, C. G., Sabirianov, R. F., Mei, W. N., Jaswal, S. S. \& Tsymbal, E. Y Interface effect on ferroelectricity at the nanoscale. Nano Lett. 6, 483487 (2006).

46. Chen, X. Q., Yamada, H., Horiuchi, T.\&Matsushige, K. Investigation of surface potential of ferroelectric organic molecules by scanning probe microscopy. Jpn. J. Appl. Phys. Part 1 38, 3932-3935 (1999).

47. Qin, M., Yao, K. \& Liang, Y. C. High efficient photovoltaics in nanoscaled ferroelectric thin films. Appl. Phys. Lett. 93, 122904 (2008).

48. Ducharme, S., Palto, S. P. \& Fridkin, V. M. in Ferroelectric and Dielectric Thin Films (ed. Nalwa, H. S.) 545-591 (Academic, 2002).

Acknowledgments - S.D. thanks the Nebraska Research Initiative and the National Science Foundation Materials Research Science and Engineering Center for financial support (DMR-0820521). A.G. acknowledges financial support from the US Department of Energy, Office of Basic Energy Sciences, Division of Materials Sciences and Engineering under award DE-SC0004530.

Author contributions - J.H. conceived the idea. J.H. and Y. Yuan designed the experiments. Y. Yuan carried out the fabrication of photovoltaic devices, the current-voltage measurement, the AFM and electrostatic force microscopy measurements and data analysis. Y. Yuan constructed the model. T.J.R., S.P. and S.D. carried out the deposition of P(VDF-TrFE) LB film and capacitance measurement. P.S. and A.G. were responsible for the PFM and conducting AFM measurement. Y. Yang provided the PSBTBT polymer. Y. Yuan, J.H., T.J.R. and S.D. analysed the data. J.H. and Y. Yuan wrote the paper. S.D. reviewed and commented on the paper. 


\section{Supplemental Materials}

\section{Efficiency enhancement in organic solar cells with ferroelectric polymers}

Yongbo Yuan ${ }^{1}$, Timothy J. Reece ${ }^{2}$, Pankaj Sharma ${ }^{2}$, Shashi Poddar ${ }^{2}$, Stephen Ducharme ${ }^{2,3}$, Alexei Gruverman ${ }^{2,3}$, Yang Yang ${ }^{4}$ and Jinsong Huang ${ }^{1,3 *}$

${ }^{1}$ Department of Mechanical Engineering, University of Nebraska, Lincoln, NE 68588

${ }^{2}$ Department of Physics and Astronomy, University of Nebraska, Lincoln, NE 68588

${ }^{3}$ Nebraska Center for Materials and Nanoscience, Lincoln NE 68583

${ }^{4}$ Department of Materials Science and Engineering, University of California- Los

Angeles, Los Angeles, CA 90095

* To whom correspondence should be addressed: jhuang2@unl.edu

\section{The induced electric field in the organic layer by ferroelectric film}

We estimated the electric field in the organic layer induced by the ferroelectric film by using a simple model of a semiconductor (S) layer and ferroelectric (FE) layer inserted between two metal electrodes $\left(\mathrm{M}_{1} / \mathrm{S} / \mathrm{FE} / \mathrm{M}_{2}\right)$, as shown in Figure $\mathrm{S} 1$. There are polarization charges (with a density of $\pm \sigma_{P}$ ) on each surface of the poled ferroelectric film. The negative polarization charges at the $\mathrm{FE} / \mathrm{M}_{2}$ interface are partially screened by the induced positive charges (with a density of $+\sigma_{S}$ ) in the metal. As a result, a net electric field penetrates into the organic layer even under short-circuit conditions, as shown in Figure S1b. Here we consider the short-circuit condition for a $M_{1} / S / F E / M_{2}$ junction. Charges can move freely between the electrodes through the external circuit so that the potential of the two electrodes is equal at infinity and the charge density at the $\mathrm{M}_{1} / \mathrm{S}$ interface is $-\sigma_{S}$ due to the charge conservation law. We apply a Thomas-Fermi model for the study of screening charges ${ }^{1,2}$. According to this model the electrical potential within metal $1(z \leq 0)$ and metal $2(z \geq L+d)$ electrode is given by: 
$\varphi_{(z)}=\left\{\begin{array}{l}\frac{\sigma_{S} \delta_{1} e^{-|z| / \delta_{1}}}{\varepsilon_{0}}, \quad z \leq 0 \\ -\frac{\sigma_{S} \delta_{2} e^{-|z-L-d| / \delta_{2}}}{\varepsilon_{0}}, \quad z \geq L+d\end{array}\right.$

here $\delta_{1}$ and $\delta_{2}$ are the Thomas-Fermi screening lengths in the $\mathrm{M}_{1}$ and $\mathrm{M}_{2}$ electrodes. The $\varphi_{(z)} \rightarrow 0$ when $z \rightarrow \pm \infty$, which is consist with the short circuit condition. The potential drop between the $\mathrm{M}_{1} / \mathrm{S}$ interface and $\mathrm{FE} / \mathrm{M}_{2}$ interface due to the electric field in the organic and ferroelectric layer is determined by:

$\varphi_{(0)}-\varphi_{(L+d)}=\frac{d\left(\sigma_{P}-\sigma_{S}\right)}{\varepsilon_{0} \varepsilon_{F E}}-\frac{L \sigma_{S}}{\varepsilon_{0} \varepsilon_{O r g}}$

here, $\varepsilon_{F E}$ and $\varepsilon_{O r g}$ is the relative dielectric constant of ferroelectric layer and organic layer, respectively. The screening charge density can be found by the using of equation (1), (2)

$\sigma_{S}=\frac{\varepsilon_{O r g} d \sigma_{P}}{\varepsilon_{F E}\left(\delta_{1}+\delta_{2}\right)+\varepsilon_{O r g} d+\varepsilon_{F E} L}$.

Finally the additional electric field $E$ in the organic layer is:

$E=\frac{\sigma_{S}}{\varepsilon_{0} \varepsilon_{O r g}}=\frac{d \sigma_{P}}{\varepsilon_{0} \varepsilon_{F E} L}$.

For an ideal condition, the one monolayer (ML) P(VDF-TrFE) on the organic layer is assumed to be continuous and uniform, with a thickness $(d)$ of $1.7 \mathrm{~nm}^{3}$. Meanwhile, the thickness of our P3HT:PCBM layer $(L)$ is about $150 \mathrm{~nm}$, the $\delta_{1}$ and $\delta_{2}$ will be less than one angstrom for "good" metals, and is negligible as compared to the thickness of the semiconducting polymer. The relative dielectric constant of $\mathrm{P}(\mathrm{VDF}-\mathrm{TrFE})$ layer $\left(\varepsilon_{F E}\right)$ is 
reported to be around $7.5^{4}$. The polarization charge density $\left(\sigma_{P}\right)$ of $\mathrm{P}(\mathrm{VDF}-\mathrm{TrFE})$ is 100 $\mathrm{mC} / \mathrm{m}^{2}$. Under this condition, the additional electric field added by the $1 \sim 3 \mathrm{ML}(\mathrm{s})$ ferroelectric $\mathrm{P}(\mathrm{VDF}-\mathrm{TrFE})$ layer in the organic semiconductor layer is found to be 17 51 $\mathrm{V} / \mu \mathrm{m}$.
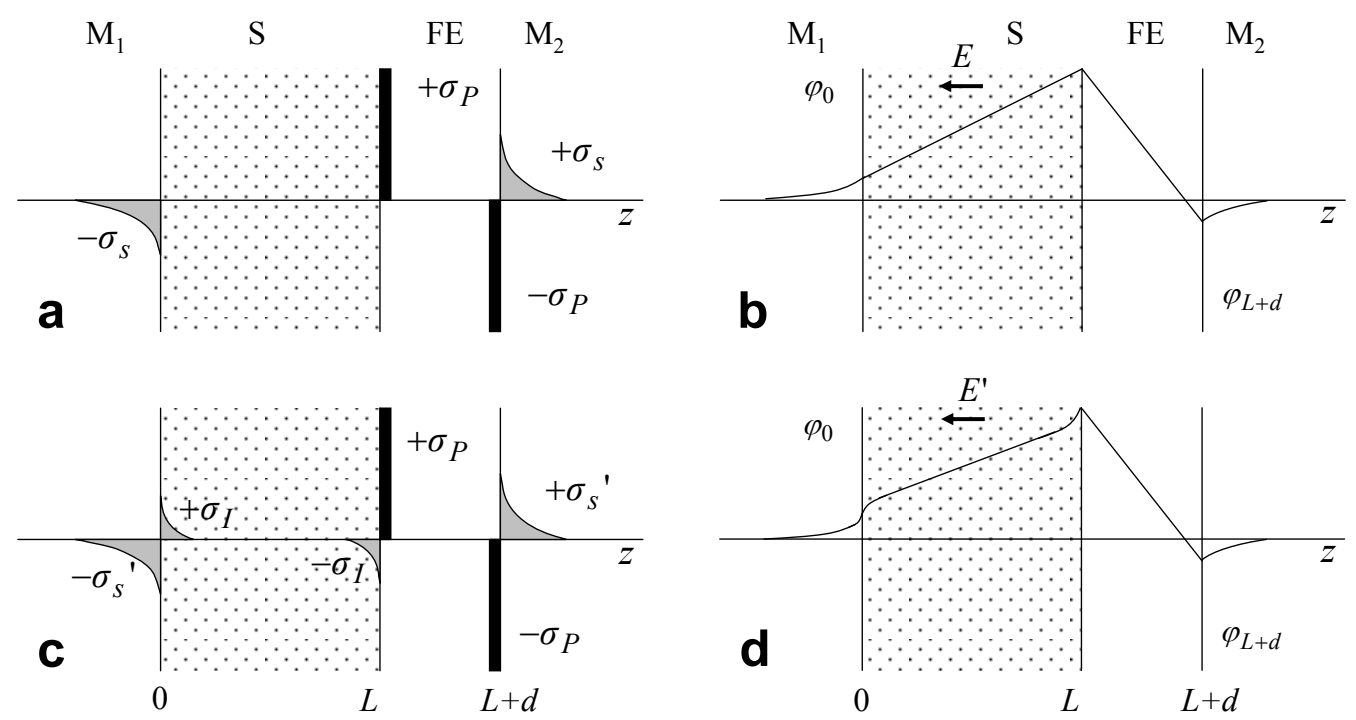

Figure S1. Electrostatics of a $\mathrm{M}_{1} / \mathrm{S} / \mathrm{FE} / \mathrm{M}_{2}$ junction. a, and $\mathbf{c}$, Charge distribution, $\mathbf{b}$, and $\mathbf{d}$, the respective electrostatic potential profile. The polarization charge density at the ferroelectric layer is $\sigma_{P}$, the induced screening charge density at the two electrodes is $\sigma_{S}$ or $\sigma_{S}{ }^{\prime}$, the trapped charges or/and mobile ions accumulated at the $\mathrm{M}_{1} / \mathrm{S}$ interface and $\mathrm{S} / \mathrm{FE}$ interface is $\sigma_{I}$.

The main concern is the possible buildup of electric charge in the cell under solar illumination. If this "space charge density" is too large, then the electric field across the cell will "collapse." We start with determining the transport time $\left(t_{T}\right)$ of an electron through the semiconductor layer under short-circuit conditions: $L(t)=\mu_{e} E t_{T}$, where $L(t)$ 
is the semiconductor layer thickness, $\mu_{\mathrm{e}}$ is an electron mobility. For a better comparison, we used the extracted average electric field $\mathrm{E}$ of $12 \mathrm{~V} / \mu \mathrm{m}$ here, which is the electric field estimated from the actual performance of ferroelectric organic photovoltaic (FE-OPV) device as discussed below. We used this transport time to roughly estimate how much the total charge of electrons builds up under solar illumination. We write $\zeta=J t_{T} / 2$, where $\zeta$ is the total electron space charge in the polymer layer (per unit area of the cell). The factor of 2 implies that the current is contributed equally by electrons and holes. For short-circuit conditions with $J_{\mathrm{SC}}=10 \mathrm{~mA} / \mathrm{cm}^{2}$, we obtain $\zeta=1.3 \times 10^{-5} \mathrm{C} / \mathrm{m}^{2}$, which is several orders magnitude less than the polarization charge density of $0.1 \mathrm{C} / \mathrm{m}^{2}$ at the ferroelectric layer surface. So the photogenerated mobile charges have negligible impact on the induced electric field by the ferroelectric layer. A more sophisticated model is constructed by considering the trapped charges or mobile ions at the $\mathrm{S} / \mathrm{FE}$ and $\mathrm{M}_{1} / \mathrm{S}$ interface, as schemed in Figure S1c. For the first glance, the net electric field in the semiconductor layer will be reduced and the influence of the poled FE layer to the semiconductor layer will be weakened. However, our calculation shows that, in practice, the trapped carriers or/and ions will be screened by the two metal electrodes and the electric field in the organic layer will remain unchanged. The detail calculation is as follows:

$$
\varphi_{(z)}=\left\{\begin{array}{l}
\frac{\sigma_{S}{ }^{\prime} \delta_{1} e^{-|z| / \delta_{1}}}{\varepsilon_{0}}, \quad z \leq 0 \\
\frac{\sigma_{S}{ }^{\prime} \delta_{1}}{\varepsilon_{0}}+\frac{\sigma_{S}{ }^{\prime}}{\varepsilon_{0} \varepsilon_{\text {Org }}} x+\varphi_{I(z)}, \quad 0 \leq z \leq L . \\
-\frac{\sigma_{S}{ }^{\prime} \delta_{2} e^{-|z-L-d| / \delta_{2}}}{\varepsilon_{0}}, \quad z \geq L+d
\end{array}\right.
$$


Where the potential $\varphi_{I(z)}$ is the potential distribution contributed by the trapped charges and ions, if present. By assuming the distribution of carriers or/and ions is exponential with a decay length of $\delta_{3}\left(\delta_{3}<<L\right)$, the potential $\varphi_{I(z)}$ can be described by Poisson's equation:

$\frac{d^{2} \varphi_{I(z)}}{d z^{2}}=-\frac{\sigma_{I}}{\varepsilon_{0} \varepsilon_{\text {Org }} \delta_{3}}\left(e^{-z / \delta_{3}}-e^{z-L / \delta_{3}}\right)$.

And similarly, the potential drop across the FE layer should satisfy boundary condition:

$\varphi_{(L)}-\varphi_{(L+d)}=\frac{\left(\sigma_{P}-\sigma_{S}{ }^{\prime}\right) d}{\varepsilon_{0} \varepsilon_{F E}}$.

Using the equation (5), (6) and (7), we arrived at the following value for the screening charge density:

$\sigma_{S}^{\prime}=\frac{\varepsilon_{O r g} d}{\varepsilon_{F E}\left(\delta_{1}+\delta_{2}\right)+\varepsilon_{F E} L+\varepsilon_{O r g} d} \sigma_{P}+\frac{\varepsilon_{F E}\left(L-2 \delta_{3}\right)}{\varepsilon_{F E}\left(\delta_{1}+\delta_{2}\right)+\varepsilon_{F E} L+\varepsilon_{O r g} d} \sigma_{I} \approx \sigma_{S}+\sigma_{I}$.

By comparing equation (8) with (6), it can be found that the screening charge density at the electrode increased when the trapped charges or mobile ions were accounted. The second item in the right side of equation (8) is induced by the trap charges or/and mobile ions. It approaches to $\sigma_{I}$ because the $\varepsilon_{F E} L$ is much larger than $\varepsilon_{O r g} d$, which means most of the trapped carriers or/and mobile ions will be screened by the electrode by add the same amount of charges in the electrode. As a result, after accounting on the space charge in the semiconductor (Figure S1c), the electric field induced in semiconductor layer is nearly unchanged from the situation excluding charge layers within the semiconductor (Figure S1a).

$E^{\prime}=\frac{\sigma_{S}{ }^{\prime}-\sigma_{I}}{\varepsilon_{0} \varepsilon_{\text {Org }}} \approx E$ 
This approximation is a good one because the charge density induced in the semiconductor $\sigma_{I}$ is generally much less than $\sigma_{P}$ because the extremely low free carrier density in organic semiconductor materials. (In the case of inorganic semiconductors, the free charge density are much larger and therefore the approximation of equation (9) is not so good ${ }^{5}$ ) This argument is supported by the capacitor measurements which show that the contribution of screening charge to the capacitance of the diodes at reverse bias is effectively negligible.

\section{The poling process of devices with ferroelectric layers}

For a better understanding of the poling process of the $\mathrm{P}(\mathrm{VDF}-\mathrm{TrFE})$ layer in the FE-OPV device, we recorded the open circuit voltage $\left(V_{\text {oc }}\right)$ of device with 1 ML P(VDFTrFE) under different poling time and temperature condition. The structure of P3HT based FE-OPV device was ITO/PEDOT (30 nm)/P3HT:PCBM (1:1 by wt, 150 $\mathrm{nm}) / \mathrm{P}(\mathrm{VDF}-\mathrm{TrFE})(1 \mathrm{ML}) / \mathrm{Al}$. As shown in Figure S2, when a bias of $15 \mathrm{~V}$ was applied continuously to the $\mathrm{Al}$ electrode at room temperature, the $V_{\mathrm{oc}}$ of devices increased slowly, it takes about one hour for the $V_{\text {oc }}$ increased from $0.476 \mathrm{~V}$ to $0.531 \mathrm{~V}$, demonstrating different degree of partial polarization. While for the device that was poled under $15 \mathrm{~V}$ at an elevated temperature of $120{ }^{\circ} \mathrm{C}$ for about $15 \mathrm{~s}$ and then field-cooled, the $V_{\mathrm{oc}}$ increased from $0.474 \mathrm{~V}$ to $0.570 \mathrm{~V}$. Heating induces a rapid and more complete poling of P(VDFTrFE) layer. The switching time of the ferroelectric layer is believed to be limited by the thermal diffusion process because the intrinsic switching time of $\mathrm{P}(\mathrm{VDF}-\mathrm{TrFE})$ layer is reported to be as short as $100 \mathrm{~ns}^{6}$. Accordingly, the poling of devices under $-2 \mathrm{~V}$ at both heating and field cooling process is referred as negative poling. We use a low bias of $-2 \mathrm{~V}$ 
applied on Al electrode for the negative poling because a higher voltage would induce too much charge injection from ITO/PEDOT:PSS, which burns the devices.

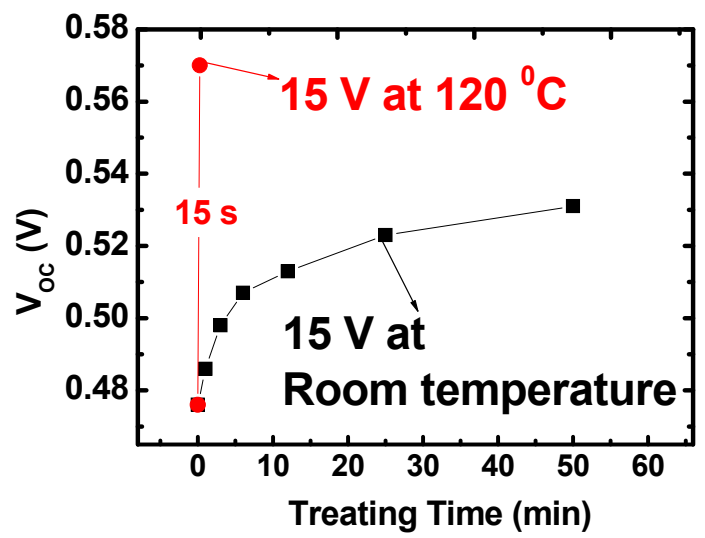

Figure S2. Polarization dynamic process of ferroelectric polymer film in the OPV device.

\section{The morphology of P(VDF-TrFE) layers}

The morphology of the 0-3 ML(s) of P(VDF-TrFE) LB film on P3HT:PCBM was studied by atomic force microscope (AFM) in tapping mode. The film roughness data are summarized in Table S1. The coating of ferroelectric polymer smoothed the semiconductor films. After annealing, the films become even more smoother. However, as shown in Figure S3, the 3 MLs P(VDF-TrFE) coated on P3HT:PCBM layer aggregates during thermal annealing and forms many nanomesas with a thickness of around $10 \mathrm{~nm}^{7}$ The aggregation of $\mathrm{P}(\mathrm{VDF}-\mathrm{TrFE})$ may derive from the mismatch of surface energy between P3HT:PCBM film and P(VDF-TrFE) film and the formation of nanomesas helps to minimize the surface energy. ${ }^{8}$ 


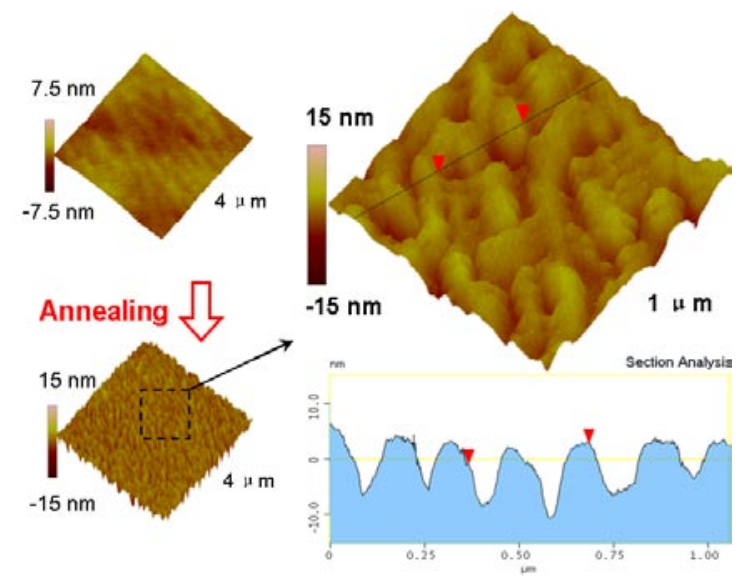

Figure S3. AFM pictures of 3 MLs of P(VDF-TrFE) films on P3HT:PCBM

Table S1. Summary of the roughness of the samples with different monolayer(s) of P(VDF-TrFE) on P3HT:PCBM , before and after annealing.

\begin{tabular}{llcccc}
\hline (PVDF-TrFE) & $0 \mathrm{ML}$ & $1 \mathrm{ML}$ & $2 \mathrm{MLs}$ & $3 \mathrm{MLs}$ \\
\hline RMS & Before Annealing & 1.72 & 1.14 & 1.25 & 0.79 \\
\cline { 2 - 2 }$(\mathrm{nm})$ & After Annealing & 1.54 & 1.16 & 0.98 & 3.59 \\
\hline
\end{tabular}

\section{Effect of the induced field on the photogenerated current of devices.}

The photogenerated current of OPV devices obtained by subtracting the dark current from the current under illumination is depend on the effective built-in electric field. The photogenerated current increases linearly near the open-circuit voltage $\left(V_{\mathrm{oc}^{-}} V<\right.$ $0.1 \mathrm{~V})$ and saturates at large reverse voltage $\left(V_{\mathrm{oc}}-V>10 \mathrm{~V}\right)$. Figure $\mathrm{S} 4$ compared the photogenerated current of positively poled FE-OPV and corresponding regular devices. The structures of the regular devices are ITO/PEDOT (30 nm)/P3HT:PC $70 \mathrm{BM} \mathrm{(1:1} \mathrm{by} \mathrm{wt,}$ $150 \mathrm{~nm}) / \mathrm{Al}$ and ITO/PEDOT $(30 \mathrm{~nm}) / \mathrm{PSBTBT}_{\mathrm{PC}} \mathrm{P}_{70} \mathrm{BM}(1.5: 1$ by $\mathrm{wt}, 90 \mathrm{~nm}) / \mathrm{Al}$, respectively. For the FE-OPVs, 1 ML P(VDF-TrFE) was inserted at the cathode interface. The photogenerated current of FE-OPV device at an applied voltage of $0 \mathrm{~V}\left(J_{\mathrm{sc}}\right)$ is equal 
to the extrapolated photocurrent of the regular device at an applied voltage of $-2.3 \mathrm{~V}$ in P3HT:PC ${ }_{70} \mathrm{BM}$ device, and $0.8 \mathrm{~V}$ in PSBTBT:PCBM device. We simply attribute the increased $J_{\text {sc }}$ in FE-OPVs to the electric field induced by the $\mathrm{P}(\mathrm{VDF}-\mathrm{TrFE})$ layer. Then the induced electric field is roughly estimated by dividing the equivalent applied voltage by the thickness of the semiconductor film, which is approximately $12 \pm 3 \mathrm{~V} / \mu \mathrm{m}(15$ $\mathrm{V} / \mu \mathrm{m}$ in $\mathrm{P} 3 \mathrm{HT}: \mathrm{PC}_{70} \mathrm{BM}$ device, and $9 \mathrm{~V} / \mu \mathrm{m}$ in $\mathrm{PSBTBT}: \mathrm{PC}_{70} \mathrm{BM}$ device).

b

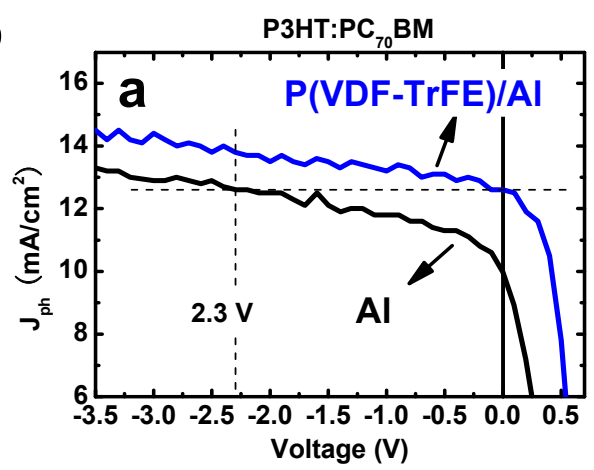

PSBTBT:PC ${ }_{70}$ BM

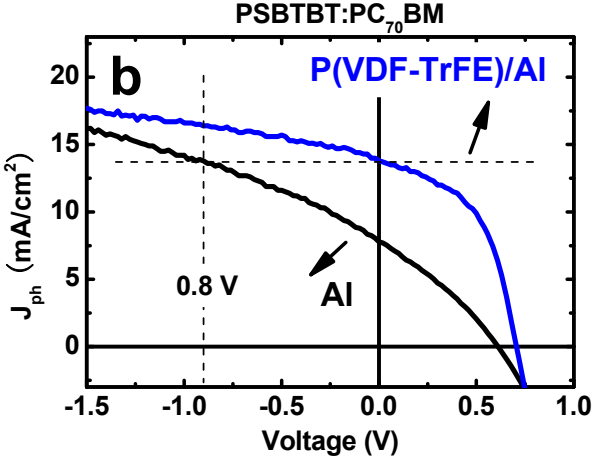

Figure S4 The photogenerated current of positive poled FE-OPV with P(VDF$\mathrm{TrFE}$ /Al cathode and regular device with $\mathrm{Al}$ cathode. The applied voltage across the semiconductor induced by the FE layer is extrapolated.

\section{Switching behaviors of the FE-OPVs}

The performance of FE-OPV devices can switch between two states repeatedly. Figure S5 show the current density-voltage (I-V) curve of OPV devices with 1 ML P(VDF-TrFE). The $J_{S C}$ of the devices switched between $11.3 \pm 0.3 \mathrm{~mA} / \mathrm{cm}^{2}$ and $7.7 \pm 0.6 \mathrm{~mA} / \mathrm{cm}^{2}$ after alternant positive poling and negative poling. Meanwhile the $V_{o c}$ and fill factor (FF) also switched evidently. After each cycle, all the output parameter came back to same levels. The repeatable switch behaviors of the devices clearly exclude the contributions of annealing effect which may 
be proposed to explain the performance improvement observed here 9

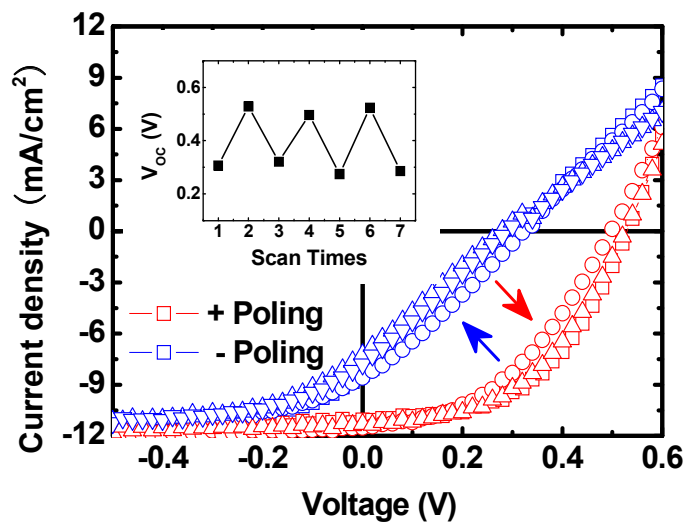

Figure S5 Switching behaviors of OPV device with $1 \mathrm{ML}$ of $\mathrm{P}(\mathrm{VDF}-\mathrm{TrFE})$ at the cathode interface

For comparison, devices without ferroelectric layer were treated under the same poling condition as the positive poling process. The performance of the devices did not show any visible improvement after poling, as shown in Figure S6. The I-V curve of the poled device was almost identical to that of unpoled device. The results exclude the contribution of other factors, such as ions movement or annealing effects ${ }^{9}$, to the efficiency improvement in FE-OPV devices.

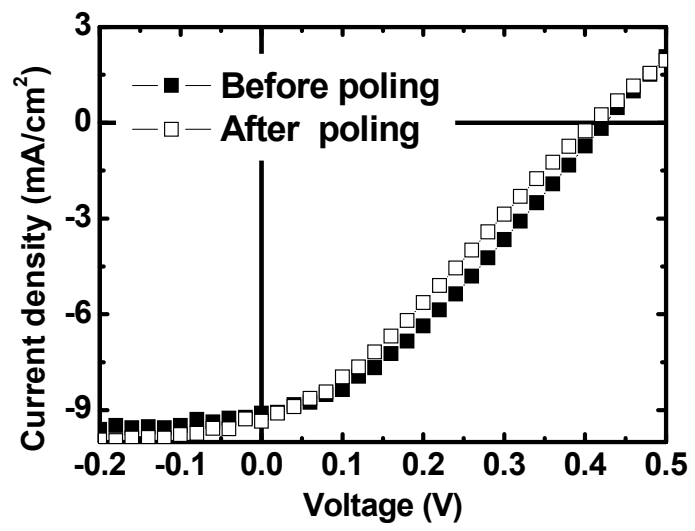


Figure S6. I-V curves of fresh regular OPV devices and the devices after positive poling.

\section{P(VDF-TrFE) layer on ITO}

In an inverted OPV device using ITO as cathode, the insertion of P(VDF-TrFE) layer also lead to improved device performance and switchable behaviors. The structure of the inverted devices is ITO/P3HT:PCBM (1:1 by wt, $150 \mathrm{~nm}) / \mathrm{MoO}_{3}(4 \mathrm{~nm}) / \mathrm{Ag}$ has an $V_{\text {oc }}$ of about $0.18 \mathrm{~V}$. A positive poling of the thin $\mathrm{P}(\mathrm{VDF}-\mathrm{TrFE})$ layer led to an improved output, and a succedent negative poling process make the performance switching back to a lower level, as shown in Figure S7, and summarized in Table S2. The results demonstrated the switching behaviors are general and independent on the electrode material used.

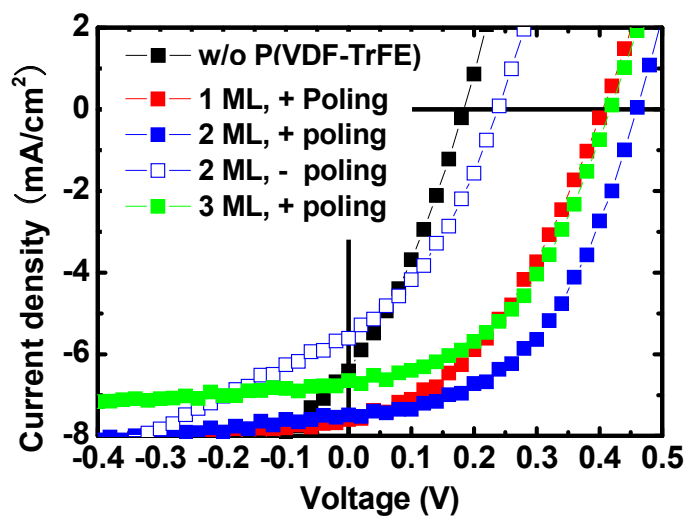

Figure S7. I-V curves of regular invert OPV devices and invert FE-OPV devices with 1-3 ML(s) of P(VDF-TrFE) on the ITO cathode. 


\section{Reference}

S1. Ashcroft, N. W. \& Mermin, N. D. Solid State Physics (Saunders College Publishing, New York, 1976).

S2. Zhuravlev, M. Y., Sabirianov, R. F., Jaswal, S. S. \& Tsymbal, E. Y. Giant electroresistance in ferroelectric tunnel junctions. Phys.Rev. Lett. 94, 246802 (2005).

S3. Bai, M. \& Ducharme, S. Ferroelectric nanomesa formation from polymer LangmuirBlodgett films. Appl. Phys. Lett. 85, 3528-3530 (2004).

S4. Ducharme, S., Palto, S. P., Blinov, L. M. \& Fridkin, V. M. Physics of two-dimensional ferroelectric polymers. AIP Conference Proceedings 535, 354-363 (2000).

S5. Reece, T. J. \& Ducharme, S. Modeling of metal-ferroelectric-insulator-semiconductor structures based on Langmuir-Blodgett copolymer films. J. Appl. Phys. 106, 124505 (2009).

S6. Ducharme, S. et al. Intrinsic ferroelectric coercive field. Phys. Rev. Letts. 84, 175-178 (1999).

S7. Bai, M. et al. Determination of the optical dispersion in ferroelectric vinylidene fluoride (70\%)/trifluoroethylene (30\%) copolymer Langmuir-Blodgett films. J. Appl. Phys. 95, 3372-3377 (2004).

S8. Li, J., Luo, Y., Bai, M. \& Ducharme, S. A continuum model on the nanomesa and nanowell formation in Langmuir-Blodgett ferroelectric polymeric films. J. Mech. Phys. Solids 54, 2162-2182 (2006).

S9. Padinger, F., Ritterger, R. S. \& Sariciftci, N. S. Effect of postproduction treatment on plastic solar cells. Adv. Funct. Mater. 13, 85-88 (2003). 